\title{
Study on the Role of Hysteroscopy in Evaluation of Infertility
}

\author{
Chitta Ranjan Nayak ${ }^{1}$, Nihar Ranjan Behera ${ }^{2}$ \\ 1Department of Obstetrics and Gynaecology, MKCG Medical College, Berhampur, Odisha, India. \\ 2Department of Obstetrics and Gynaecology, SCB Medical College, Cuttack, Odisha, India.
}

\section{ABSTRACT}

\section{BACKGROUND}

The burden of infertility is on the rise and there has been a constant effort to improve and refine our diagnostic and treatment approach to the patients. Today most of the clinicians accept that complete infertility workup should include evaluation of the uterine cavity and many investigators consider hysteroscopy as the gold standard for this purpose. It is however a fact that it is still not widely used as a routine diagnostic procedure in the initial workup of infertile women by majority of the care givers. We wanted to evaluate the role of hysteroscopy in detecting the uterine factors associated with infertility.

\section{METHODS}

A prospective observational study was conducted in the department of obstetrics and gynaecology of SCB medical college, Cuttack from March 2018 to March 2019. One hundred infertile women were selected as study sample after considering the inclusion and exclusion criteria. Diagnostic hysteroscopy was performed in all with $5 \mathrm{~mm}$ 30-degree telescope.

\section{RESULTS}

It was possible to get a panoramic and magnified view of the endocervical canal, uterine cavity, cornu and ostia. Several uterine factors could be detected in as many as $15 \%$ of primary and $33 \%$ of secondary infertility cases. Most common pathologies detected were polyps, submucous myomas, septum, cornual fibrosis and intra uterine adhesions. The procedure was found to be quite informative, safe, easy to perform, acceptable to the study participants and without any major complications.

\section{CONCLUSIONS}

Hence, we could arrive at a conclusion that the initial evaluation of any infertile women should include diagnostic hysteroscopy which can accurately detect the uterine factors responsible for the inability to conceive and subsequently can improve the results of treatment.

\section{KEY WORDS}

Infertility, Initial Workup, Uterine Factors, Hysteroscopy
Corresponding Author: Dr. Nihar Ranjan Behera, G-102, Utkal Vatika, Jharpada Canal Road, PS-Laxmi Sagar, Bhubaneswar-751006, Odisha, India. E-mail: nihar_beheral18.5@rediffmail.com

DOI: $10.14260 /$ jemds/2020/415

Financial or Other Competing Interests: None.

How to Cite This Article: Nayak CR, Behera NR. Study on the role of hysteroscopy in evaluation of infertility. J. Evolution Med. Dent. Sci. 2020;9(26): 1908-1911, DOI:
Submission 24-03-2020,

Peer Review 28-05-2020, Acceptance 05-06-2020, Published 29-06-2020. 


\section{BACKGROUND}

Infertility is defined as the inability to conceive after one year of regular unprotected intercourse. Today it affects $15 \%$ of couples in the reproductive age group. ${ }^{1}$ Prevalence of this problem is increasing day by day due to several reasons like deferral of age for childbearing due to career reasons, change in attitude towards family and raising children, increase in sexually transmissible diseases and increase in the number of young women with polycystic ovarian diseases. It is a condition with profound psychological and emotional impacts. Most couples look upon infertility as a life crisis in which they feel isolated and helpless. Frustration, anger, depression, grief, guilt, anxiety and poor self-esteem are the challenges they face. ${ }^{2}$

Nevertheless, evaluation of such a case and arriving at a correct etiological diagnosis is becoming more and more challenging for the gynaecologists. Though the main factors leading to infertility are known and they are anovulation (20\%), male factors $(25 \%)$, pelvic $(30 \%)$, uterine $(10 \%)$ in about $15 \%$ of cases the cause remain unexplained. They are missed by the basic diagnostic procedures like semen analysis, follicular study, hysterosalpingography, ultrasonography and laparoscopy. In reality these methods fail to evaluate the inside of the uterine cavity adequately. At this juncture hysteroscopy as a diagnostic and therapeutic modality is gaining popularity. It has got several advantages like wider availability, acceptability, safety and most importantly its ability to directly visualize the inside of the uterine cavity. ${ }^{3}$

Several researchers do claim that hysteroscopy is a far more superior diagnostic tool which allows accurate localization of pathologies, targeted sampling of lesions and precise correction of defects like polyps, adhesions, submucous myomas, septums and proximal tubal blocks. It can be done as an office procedure or day-care surgery without need for hospitalization or anaesthesia. Furthermore, it can be combined with laparoscopy as a single procedure in the investigation of infertility patients. ${ }^{4}$

There are studies which have compared hysteroscopy with hysterosalpingography in relation to infertility and found hysteroscopy to be a more promising and novel technique. ${ }^{5}$ Literature also suggests that many patients who had normal findings in ultrasonography were subsequently found to have intrauterine pathologies like polyps, septum, submucous myomas and adhesions in hysteroscopy. ${ }^{6}$ While all these are pointing finger towards hysteroscopy as valuable first line tool in infertility workup many still do not accept it. With this background in mind we wanted to address the question - whether diagnostic hysteroscopy is to be included in the basic investigation protocol for cases of primary and secondary infertility. A clear and definite answer to this question will go a long way to help the childless couples in the general population.

We wanted to evaluate the role of hysteroscopy in detecting the uterine factors associated with infertility and determine as to whether it is an easy to perform, safe, less time consuming and its acceptability by the patients.

\section{METHODS}

This was a cross sectional study undertaken from March 2018 to March 2019 in the department of Obstetrics and Gynaecology, SCB Medical College, Cuttack.

\section{Inclusion Criteria}

Women with primary and secondary infertility attending Gynaec OPD and admitted for evaluation.

\section{Exclusion Criteria}

Women with ovulatory factors, tubal factors and other diagnosed extrauterine causes.

- Women with active PID

- Women who had already been worked up with hysteroscopy and with normal report.

- Women who became unfit for anaesthesia and women who did not turn up on the day of procedure.

One hundred women who fulfilled the selection criteria were included in the study sample. The calculation of sample size was based on the review of literature. All participants were explained the purpose of the study and details of the procedure they will undergo along with their risks and benefits before obtaining written consent. After detailed history taking and clinical examination preoperative evaluation was conducted as per the standard protocol before any surgery under general anaesthesia. The participants were admitted in the morning of surgery in empty stomach. After anaesthesia patients were placed in lithotomy position, painted and draped. Bladder was emptied with a catheter by sterile technique. Bimanual examination was performed to know the position and size of uterus. Cervical canal was dilated to number 6 Hegar's. $4 \mathrm{~mm}$ hysteroscope with 30degree oblique lens was introduced. Normal saline was used as distension medium along with halogen light source. First the endocervical canal and then the endometrial cavity including both cornu and ostia was visualized. Any lesions like polyps, myomas, septum and adhesions were noted. As the cervical canal distends the endocervical canal shows longitudinal folds, papilla and clefts. The vascular pattern of endocervix reveals branching tree like vessels normally. The endometrium appears pink white in colour because the patients were posted in the secretory phase. The gland opening appear as white ringed elevation surrounded by net like vessels. Mucous polyps appeared as single or multiple sessile or pedunculated with colour same as that of the surrounding endometrium. Fibroid polyps were smooth and appeared paler than the endometrium. They were seen to move in streaming movements in the liquid medium. The scope can hit and displace the polyp. This helped to differentiate it from a myoma. The myomas protrude into uterine cavity to a varying degree. They are not covered by endometrium and are yellow white in colour. Often the surface is traversed by blood vessels and they are firm with little mobility when depressed by telescope. Intrauterine synechiae (Asherman's syndrome) appeared as multiple thick and thin fibrous bands of tissue inside uterine cavity. A complete septum could also be seen and differentiated from a 
bicornuate uterus. In septate uterus fundus was wide but in bicornuate uterus fundus was heart shaped. All these findings as and when found were recorded for future analysis. Observation was also made regarding the time consumed from start of the procedure to end. The difficulties encountered such as issues of dilatation of cervix, negotiation of scope, visualization and insufflation were also listed. Intraoperative and postoperative complications related to both anaesthesia and the procedure were also noted. The overall satisfaction of the participants and acceptance of the procedure was assessed at the end by means of a validated questionnaire.

\section{Statistical Analysis}

Statistical analysis was done using IBM SPSS Statistics software.

\section{RESULTS}

\begin{tabular}{|ccccc|}
\hline Age in Years & Primary & Percentage & Secondary & Percentage \\
$20-24$ & 16 & 42.10 & 9 & 14.51 \\
$25-29$ & 7 & 18.42 & 18 & 29.03 \\
$30-34$ & 9 & 23.68 & 22 & 35.48 \\
$35-40$ & 6 & 15.78 & 13 & 20.96 \\
Total & $\mathbf{3 8}$ & $\mathbf{1 0 0}$ & $\mathbf{6 2}$ & $\mathbf{1 0 0}$ \\
Mean & 27.26 & \multicolumn{4}{c|}{} \\
Median & 25.5 & 30.90 & \\
\hline \multicolumn{5}{c}{ Table 1. Distribution of Cases According to Age } \\
\hline
\end{tabular}

\begin{tabular}{|cccccc|}
\hline $\begin{array}{c}\text { Duration of } \\
\text { Infertility in Years }\end{array}$ & Primary & $\mathbf{\%}$ & Secondary & $\mathbf{\%}$ & Total \% \\
$1-3$ & 21 & 55.26 & 50 & 80.64 & 71 \\
$4-6$ & 12 & 31.57 & 12 & 19.35 & 24 \\
$7-10$ & 5 & 13.15 & - & - & 5 \\
Total & $\mathbf{3 8}$ & $\mathbf{1 0 0}$ & $\mathbf{6 2}$ & $\mathbf{1 0 0}$ & $\mathbf{1 0 0}$ \\
Mean & 3.8 & \multicolumn{5}{c|}{} \\
Median & 3 & 2 & & \\
\hline Table 2. Distribution of Cases According to Duration of Infertility &
\end{tabular}

\begin{tabular}{|ccc|}
\hline Time Taken in Minutes & Number of Women & \% of Women \\
$15-20$ & 24 & 24 \\
$21-25$ & 39 & 39 \\
$26-30$ & 37 & 37 \\
Mean = 22.44 minutes & \\
Median = 24 minutes & \\
\hline \multicolumn{2}{|c|}{ Table 3. Time Taken for the Procedure } \\
\hline
\end{tabular}

\begin{tabular}{|cccccc|}
\hline $\begin{array}{c}\text { Hysteroscopic } \\
\text { Findings }\end{array}$ & $\begin{array}{c}\text { Primary } \\
\text { Infertility }\end{array}$ & $\mathbf{\%}$ & Secondary & $\mathbf{\%}$ & Total \% \\
Cervical polyp & 1 & 6.66 & 1 & 3.03 & 2 \\
Cervical stenosis & - & - & 3 & 9.09 & 3 \\
Distorted cavity & - & - & 1 & 3.03 & 1 \\
Uterine synechiae & - & - & 7 & 21.21 & 7 \\
Septate Uterus & 4 & 26.66 & 3 & 9.09 & 7 \\
Bicornuate uterus & 3 & 20 & 4 & 12.12 & 7 \\
Submucous myoma & 3 & 20 & 6 & 18.18 & 9 \\
Endometrial polyp & 2 & 13.33 & 7 & 21.21 & 9 \\
Cornual fibrosis & 1 & 6.66 & 1 & 3.03 & 2 \\
Bilateral corneal & 1 & 6.66 & - & - & 1 \\
fibrosis & $\mathbf{1 5}$ & $\mathbf{1 0 0}$ & $\mathbf{3 3}$ & $\mathbf{1 0 0}$ & $\mathbf{4 8}$ \\
\hline Total & Table 4. Hysteroscopic Findings \\
\hline \multicolumn{7}{|l}{} \\
\hline
\end{tabular}

\begin{tabular}{|ccc|}
\hline Complications & Total Number & Percentage \\
Pain lower abdomen & 12 & 12 \\
Bleeding PV & 4 & 4 \\
Pyrexia & 1 & 1 \\
Backache & - & - \\
Nausea \& vomiting & - & - \\
Fainting attack & - & - \\
Parietal emphysema & - & - \\
Injury to bowl & - & - \\
Injury to bladder & - & - \\
Uterine perforation & - & - \\
Anaesthesia complications & - & - \\
Total & $\mathbf{1 7}$ & $\mathbf{1 7}$ \\
\hline Table 5. Complications during and after the Procedure \\
\hline
\end{tabular}

Out of the one hundred cases 38 (38\%) were cases of primary infertility and $62(62 \%)$ were cases of secondary infertility. Majority of women with primary infertility belonged to the age group of 20-24 years and that of secondary infertility belonged to $30-34$ years age group. In our study uterine factors were associated in $48 \%$ of cases. Uterine pathology was more frequent in secondary (33\%) than primary infertility (15\%). Most common uterine pathology were endometrial polyps, submucous myomas, intrauterine adhesions. The time taken for the procedure excluding anaesthesia ranged from 15 to 30 minutes with a mean duration of 22.44 minutes and median of 24 minutes. There were no major complications in any of the women who underwent the procedure. Minor complications like mild bleeding PV and lower abdominal pain and transient fever was there in 17 cases. Difficulties encountered were minimal which included five cases where cervix was rigid, six cases where negotiation of scope was a problem due to acute anteversion of uterus, four cases with secondary infertility with cervical tear resulting in back flow of normal saline, three cases of visualization issues due to adhesions and only one case in which false passage resulted due to undiagnosed deviated uterus. $98 \%$ participants responded to the question are saying that they were happy with the procedure and they had no regrets.

\section{DISCUSSION}

The results show that as many as $48 \%$ of study participants had some abnormal hysteroscopy findings. Similar results were obtained in the study by Jesmine Banu et al ${ }^{7}$ where $49 \%$ patients had uterine pathology. Similarly, a retrospective study by Cenksoy P et al ${ }^{8}$ in 2013 showed $44.9 \%$ women with infertility had abnormal hysteroscopic findings. Such a high figure of $48 \%$ for intrauterine pathologies in our study and other studies was obtained due to the fact that we had selected the cases where the ovulatory factors and tubal factors were excluded. So, we had to concentrate on those cases where ovulation was normal, and tubes were patent and so the likely hood of detecting intrauterine factors was high. Nevertheless, in doing so it became clearly evident that intrauterine factors are quite common, and hysteroscopy is the best method of detecting them. If hysteroscopy would have been not done in these cases, then we would not have diagnosed these causes in them. Then they would have been wrongly stamped as cases of unexplained infertility. We have not excluded cases with abnormal semen tests keeping in view the chances of combined factors in the partners. In the present study it was found that hysteroscopy as a procedure could be easily performed in a few minutes without encountering much difficulties. There were minimal complications associated with this technique. Few participants had only mild pain in the lower abdomen which subsided with analgesics. Minimal bleeding per vagina was not significant and post-operative pyrexia was reactionary which subsided after 24 hours. The level of acceptance and satisfaction of the patients was very high at the end of the day. 


\section{CONCLUSIONS}

It was quite evident from our study that uterine pathologies are not uncommon in infertile women. These pathologies can be easily and accurately diagnosed by hysteroscopy. The procedure is quite safe and less time consuming. The greatest benefit is a direct visualization and a panoramic magnified view of cervical canal, uterine cavity, cornu and ostia. ${ }^{9}$ Few years back diagnostic laparoscopy was not included in the initial workup of infertile women. Today no investigation protocol of infertility is complete without a laparoscopy. ${ }^{10}$ Similar is the merit of hysteroscopy and it is considered by many as far more superior than hysterosalpingography for evaluation of uterine cavity and associated lesions. ${ }^{11}$ It can also be performed simultaneously with laparoscopy as a combined procedure. Besides, it has also got the therapeutic advantage because many pathologies like polyps, submucous myomas, adhesions and proximal tubal blocks can be simultaneously treated in the same sitting. Hence, we can conclude that the initial workup of infertile couple should include hysteroscopic evaluation along with other basic investigations like seminal analysis, ovulation study, tubal patency test and diagnostic laparoscopy. We sincerely believe that if gynaecologists value and accept the usefulness of this novel diagnostic procedure, then they will not only get enormous benefit but also pass it on to the society at large.

\section{REFERENCES}

[1] Padubidri VG, Daftary SN. Shaw's text book of gynaecology. $13^{\text {th }}$ edn. Reed Eisevier India Pvt Ltd 2004: p. 198.
[2] Daftary SN, Patki A. Reproductive endocrinology and infertility. Chap- 20. BI Publications Pvt Ltd 2009:20018.

[3] Corfman RS. Indications for hysteroscopy. Obstet Gynecol Clin North Am 1988;15(1):41-9.

[4] Balmaceda JP, Ciuffardi I. Hysteroscopy and assisted reproductive technology. Obstet Gynecol Clin North Am 1995;22(3):507-18.

[5] Vujovic M, Garalejic E, Arsic B, et al. Hysterosalpingography versus hysteroscopy in intrauterine pathology research of infertile patients. Clin Exp Obstet Gynecol 2015;42(2):141-5.

[6] Yucebilgin MS, Aktan E, Bozkurt K, et al. Comparison of hydrosonography and diagnostic hysteroscopy in the evaluation of infertile patients. Clin Exp Obstet Gynecol 2004;31(1):56-8.

[7] Banu J, Deeba F, Fatima P, et al. Evaluation of uterine cavity by hysteroscopic examination in infertile women in BSMMU. Bangabandhu Sheikh Mujib Medical University Journal 2016;9(1):32-7.

[8] Cenksoy P, Ficicioglu C, Yıldırım G, et al. Hysteroscopic findings in women with recurrent IVF failures and the effect of correction of hysteroscopic findings on subsequent pregnancy rates. Arch Gynecol Obstet 2013;287(2):357-60.

[9] Malhotra N, Sood M. Role of hysteroscopy in infertile women. J Indian Med Assoc 1997;95(9):499-525.

[10] Berek JS, Rinehart RD, Hillard PA, et al. Berek and Novak's gynecology. $15^{\text {th }}$ edn. Lippincott Williams \& Wilkins 2012:973-4.

[11] Ragni G, Lombroso GC, Bestetti 0, et al. Hysteroscopy versus hysterosalpingography in infertile patients. Int J Fertil 1984;29(3):141-2. 